\title{
On the Jump set of Solutions of the Total Variation Flow
}

\author{
V. Caselles (*) - K. Jalalzai (**) - M. Novaga (***)
}

Abstract - We show that the jump set of the solution of the minimizing Total Variation flow decreases with time for any initial condition in $B V(\Omega) \cap L^{N}(\Omega)$. We prove that the size of the jump also decreases with time.

Mathematics Subject Classification (2010). 35J20, 94A08, 26B30, 68U10

KEYwords. Total variation flow, demonising model, nonlinear parabolic equations, functions of bounded variation

\section{Introduction}

The use of total variation as a regularization tool for image denoising and restoration was introduced by L. Rudin, S. Osher and E. Fatemi in [19]. If $\Omega$ denotes the image domain, the Total Variation denoising problem corresponds to solving the minumum problem

$$
\min _{u \in B V(\Omega)}\left\{\int_{\Omega}|D u|+\frac{1}{2 \lambda} \int_{\Omega}(u-f)^{2} d x, \quad \lambda>0\right\} .
$$

One of the main features of (1), confirmed by numerical simulations, is its ability to restore the discontinuities of the image $[19,14,16]$. The a

(*) Indirizzo dell'A.: Departament de Tecnologia, Universitat Pompeu-Fabra, Barcelona, Spain.

E-mail: vicent.caselles@upf.edu

(**) Indirizzo dell'A.: CMAP, CNRS UMR 7641, Ecole Polytechnique, 91128 Palaiseau, France.

E-mail: khalid.jalalzai@polytechnique.edu

(***) Indirizzo dell'A.: Dipartimento di Matematica, Università di Padova, Via Trieste 63, 35121 Padova, Italy.

E-mail:novaga@math.unipd.it 
priori assumption is that functions of bounded variation (the $B V$ model [3]) are a reasonable functional setting for many problems in image processing and, in particular, for denoising and restoration. Typically, functions of bounded variation admit a set of discontinuities which is countably rectifiable [3], being continuous (in the measure theoretic sense) away from discontinuities. The discontinuity set corresponds to the edges in the image, and the ability of Total Variation regularization to recover edges is one of the main features which advocates for the use of this model in image processing (its ability to describe textures is less clear, even if some textures can be recovered up to a certain scale of oscillation).

As a support to this idea, in [10] (see also [13, 12]) it has been proved that the jump set of the solution $u$ of the TV denoising problem (1) is contained in the jump set of the datum $f$, assuming that $f \in B V(\Omega) \cap L^{\infty}(\Omega)$. Moreover the size of the jump of $u$ at any point $x$ in its jump set is bounded by the size of the jump of $f$. This result was explicitly stated in [18] in a more general context, even if for the Total Variation it is essentially contained in [10] (see also [12]).

The purpose of this paper is to prove the corresponding result for solutions of the the minimizing Total Variation flow, with Neumann or Dirichlet boundary conditions, and for the Cauchy problem in $\mathbb{R}^{N}$. That is, if $u(t)$ is the solution of the TV flow and $J_{u(t)}$ denotes the jump set of $u(t)$, we prove that

$$
J_{u(t)} \subseteq J_{u(s)} \subseteq J_{u(0)} \quad \mathcal{H}^{N-1} \text { a.e. for any } t>s>0 .
$$

Moreover, letting $[u(t)]$ be the jump size of $u(t)$, we also show that

$$
[u(t)] \leq[u(s)] \quad \mathcal{H}^{N-1} \text { a.e. on } J_{u(t)} \text { for any } t \geq s \geq 0 .
$$

The inclusions in (2) have already been proved in [10], under the assumption that $u_{0}$ belongs to the domain of the operator $-\operatorname{div}\left(\frac{D u}{|D u|}\right)$ in $L^{\infty}(\Omega)$. Some sufficient conditions for this to happen were given in [10]. By exploting (3), in the present paper we get rid of this condition on $u(0)$.

Let us finally describe the plan of the paper. In Section 2 we recall some basic facts about functions of bounded variation that will be used in the sequel. In Section 3 we review the result of [10] on the jump of the solutions of the denoising problem (1), explicitly proving that the size of the jump of the solution is bounded by the size of the jump of the initial datum. Finally, in Section 4 we extend this result to the Total Variation flow. 


\section{Notation and preliminaries on $B V$ functions}

Let $\Omega$ be an open subset of $\mathbb{R}^{N}$. A function $u \in L^{1}(\Omega)$ whose gradient $D u$ in the sense of distributions is a (vector valued) Radon measure with finite total variation in $\Omega$ is called a function of bounded variation. The class of such functions will be denoted by $B V(\Omega)$. The total variation of $D u$ on $\Omega$ turns out to be

$$
\sup \left\{\int_{\Omega} u \operatorname{div} z d x: z \in C_{0}^{\infty}\left(\Omega ; \mathbb{R}^{N}\right),|z(x)| \leq 1 \forall x \in \Omega\right\},
$$

(where for a vector $v=\left(v_{1}, \ldots, v_{N}\right) \in \mathbb{R}^{N}$ we set $\left.|v|^{2}:=\sum_{i=1}^{N} v_{i}^{2}\right)$ and will be denoted by $|D u|(\Omega)$ or by $\int_{\Omega}|D u|$. The map $u \rightarrow|D u|(\Omega)$ is $L_{\text {loc }}^{1}(\Omega)$-lower semicontinuous. $B V(\Omega)$ is a Banach space when endowed with the norm $\|u\|:=\int_{\Omega}|u| d x+|D u|(\Omega)$.

A measurable set $E \subseteq \Omega$ is said to be of finite perimeter in $\Omega$ if (4) is finite when $u$ is substituted with the characteristic function $\chi_{E}$ of $E$. The perimeter of $E$ in $\Omega$ is defined as $P(E, \Omega):=\left|D \chi_{E}\right|(\Omega)$. We denote by $\mathcal{L}^{N}$ and $\mathcal{H}^{N-1}$, respectively, the $N$-dimensional Lebesgue measure and the $(N-1)$-dimensional Hausdorff measure in $\mathbb{R}^{N}$.

Let $u \in\left[L_{\mathrm{loc}}^{1}(\Omega)\right]^{m}$. We say that $u$ has approximate limit at $x \in \Omega$ if there exists $z \in \mathbb{R}^{m}$ such that

$$
\lim _{\rho \downarrow 0} \frac{1}{|B(x, \rho)|} \int_{B(x, \rho)}|u(y)-z| d y=0 .
$$

The set of points where this does not hold is called the approximate discontinuity set of $u$, and is denoted by $S_{u}$. Using Lebesgue's differentiation theorem, one can show that the approximate limit $z$ exists at $\mathcal{L}^{N}$-a.e. $x \in \Omega$, and is equal to $u(x)$ : in particular, $\left|S_{u}\right|=0$.

If $x \in \Omega \backslash S_{u}$, the vector $z$ is uniquely determined by (5) and we denote it by $\tilde{u}(x)$. We say that $u$ is approximately continuous at $x$ if $x \notin S_{u}$ and $\tilde{u}(x)=u(x)$, that is if $x$ is a Lebesgue point of $u$ with respect to the Lebesgue measure.

For $u \in B V(\Omega)$, the gradient $D u$ is a Radon measure that decomposes into its absolutely continuous and singular parts $D u=D^{a} u+D^{s} u$. Then $D^{a} u=\nabla u d x$ where $\nabla u$ is the Radon-Nikodym derivative of the measure $D u$ with respect to the Lebesgue measure in $\mathbb{R}^{N}$. The function $u$ is approximately differentiable $\mathcal{L}^{N}$ a.e. in $\Omega$ and the approximate differential 
coincides with $\nabla u(x) \mathcal{L}^{N}$ a.e. The singular part $D^{s} u$ can be also split in two parts: the jump part $D^{j} u$ and the Cantor part $D^{c} u$. We say that $x \in \Omega$ is an approximate jump point of $u$ if there exist $u^{+}(x) \neq u^{-}(x) \in \mathbb{R}$ and $\left|v_{u}(x)\right|=1$ such that

$$
\begin{aligned}
& \lim _{\rho \downarrow 0} \frac{1}{\left|B_{\rho}^{+}\left(x, v_{u}(x)\right)\right|} \int_{B_{\rho}^{+}\left(x, v_{u}(x)\right)}\left|u(y)-u^{+}(x)\right| d y=0 \\
& \lim _{\rho \downarrow 0} \frac{1}{\left|B_{\rho}^{-}\left(x, v_{u}(x)\right)\right|} \int_{B_{\rho}^{-}\left(x, v_{u}(x)\right)}\left|u(y)-u^{-}(x)\right| d y=0,
\end{aligned}
$$

where

$$
\begin{aligned}
& B_{\rho}^{+}\left(x, v_{u}(x)\right)=\left\{y \in B(x, \rho):\left\langle y-x, v_{u}(x)\right\rangle>0\right\} \\
& B_{\rho}^{-}\left(x, v_{u}(x)\right)=\left\{y \in B(x, \rho):\left\langle y-x, v_{u}(x)\right\rangle<0\right\} .
\end{aligned}
$$

We denote by $J_{u}$ the set of approximate jump points of $u$. If $u \in B V(\Omega)$, the set $S_{u}$ is countably $\mathcal{H}^{N-1}$ rectifiable, $J_{u}$ is a Borel subset of $S_{u}$ and $\mathcal{H}^{N-1}\left(S_{u} \backslash J_{u}\right)=0$ (see [3]). In particular, we have that $\mathcal{H}^{N-1}$-a.e. $x \in \Omega$ is either a point of approximate continuity of $\tilde{u}$, or a jump point with two limits in the above sense. Eventually, we have

$$
D^{j} u=D^{s} u\left\llcorner J_{u}=\left(u^{+}-u^{-}\right) v_{u} \mathcal{H}^{N-1}\left\llcorner J_{u} \quad \text { and } D^{c} u=D^{s} u\left\llcorner\left(\Omega \backslash S_{u}\right) .\right.\right.\right.
$$

For $x \in J_{u}$ we set $[u(x)]=u^{+}(x)-u^{-}(x)$.

For a comprehensive treatment of functions of bounded variation we refer to [3].

\section{The jump set of solutions of the TV denoising problem}

Given a function $f \in L^{2}(\Omega)$ and $\lambda>0$ we consider the minimum problem

$$
\min _{u \in B V(\Omega)} \int_{\Omega}|D u|+\frac{1}{2 \lambda} \int_{\Omega}(u-f)^{2} d x=: \mathcal{F}_{\lambda}(u) .
$$

Notice that problem (6) always admits a unique solution $u_{\lambda}$, since the functional $\mathcal{F}_{\lambda}$ is strictly convex.

As we mentioned in the Introduction, one of the main reasons to introduce the Total Variation as a regularization term in imaging problems is its ability to recover the discontinuities of the function $f$. In this section we recall a result proved in [10] showing that the jump set of $u_{\lambda}$ is always 
contained in the jump set of $f$, that is, the model (6) does not create any new discontinuity besides the existing ones. We refine the proof given in [10] proving that the size of the jump decreases. This fact was observed in [18] where it was proved in the more general context of weighted total variation.

Let us recall the following Proposition, which is proved in $[15,1]$.

Proposition 3.1. Let $u_{\lambda}$ be the (unique) solution of (6). Then, for any $t \in \mathbb{R},\left\{u_{\lambda}>t\right\}$ (respectively, $\left\{u_{\lambda} \geq t\right\}$ ) is the minimal (resp., maximal) solution of the minimal surface problem

$$
\min _{E \subseteq \Omega} P(E, \Omega)+\frac{1}{\lambda} \int_{E}(t-f(x)) d x .
$$

In particular, for all $t \in \mathbb{R}$ but a countable set, $\left\{u_{\lambda}=t\right\}$ has zero measure and the solution of (7) is unique (up to a negligible set).

A proof that $\left\{u_{\lambda}>t\right\}$ and $\left\{u_{\lambda} \geq t\right\}$ both solve (7) is found in [15, Prop. 2.2]. The proof of Proposition 3.1 then follows from the co-area formula and from the following comparison result for solutions of (7) (see [1, Lemma 4]):

LEMMA 3.2. Let $f, g \in L^{1}(\Omega)$ and $E$ and $F$ be respectively minimizers of

$$
\min _{E} P(E, \Omega)-\int_{E} f(x) d x \text { and } \min _{F} P(F, \Omega)-\int_{F} g(x) d x .
$$

Then, if $f<g$ a.e., $|E \backslash F|=0$ (in other words, $E \subseteq F$ up to a negligible set).

From Proposition 3.1 and the regularity theory for surfaces of prescribed curvature (see for instance [2]), one has the following regularity result.

Corollary 3.3. Let $f \in L^{p}(\Omega)$, with $p>N$. Then, for all $t \in \mathbb{R}$ the super-level set $E_{t}:=\left\{u_{\lambda}>t\right\}$ (respectively, $\left\{u_{\lambda} \geq t\right\}$ ) has boundary of class $C^{1, \alpha}$, for all $\alpha<(p-N) / p$, out of a closed singular set $\Sigma$ of Hausdorff dimension at most $N-8$. Moreover, if $p=\infty$, the boundary of $E_{t}$ is of class $W^{2, q}$ out of $\Sigma$, for all $q<\infty$, and is of class $C^{1,1}$ if $N=2$.

Before stating the main result of this section, we recall two simple Lemmata (see [11]). 
Lemma 3.4. Let $U$ be an open set in $\mathbb{R}^{N}$ and $v \in W^{2, p}(U), p \geq 1$. We have that

$$
\operatorname{div}\left(\frac{\nabla v}{\sqrt{1+|\nabla v|^{2}}}\right)=\operatorname{trace}\left(A(\nabla v) D^{2} v\right) \quad \text { a.e. in } U,
$$

where

$$
A(\xi)_{i j}:=\frac{1}{\left(1+|\xi|^{2}\right)^{\frac{1}{2}}}\left(\delta_{i j}-\frac{\xi_{i} \xi_{j}}{\left(1+|\xi|^{2}\right)}\right) \quad \xi \in \mathbb{R}^{N} .
$$

LEMMA 3.5. Let $U$ be an open set in $\mathbb{R}^{N}$ and $v \in W^{2,1}(U)$. Assume that $u$ has a minimum at $y_{0} \in U$ and

(8) $\lim _{\rho \rightarrow 0+} \frac{1}{\left|B\left(y_{0}, \rho\right)\right|}$

$\int_{B\left(y_{0}, \rho\right)} \frac{\left|u(y)-u\left(y_{0}\right)-\nabla u\left(y_{0}\right) \cdot\left(y-y_{0}\right)-\frac{1}{2}\left\langle D^{2} v\left(y_{0}\right)\left(y-y_{0}\right), y-y_{0}\right\rangle\right|}{\rho^{2}} d y=0$,

then $D^{2} v\left(y_{0}\right) \geq 0$.

Recall that, if $v \in W^{2,1}(U)$, then (8) holds a.e. in $U$ [20, Th. 3.4.2].

Theorem 1. Let $f \in B V(\Omega) \cap L^{\infty}(\Omega)$. Then, for all $\lambda>0$,

$$
J_{u_{\lambda}} \subseteq J_{f}
$$

up to a set of zero $\mathcal{H}^{N-1}$-measure. Moreover

$$
\left[u_{\lambda}(x)\right] \leq[f(x)] \quad \mathcal{H}^{N-1} \text {-a.e. on } J_{u_{\lambda}} .
$$

Proof. Notice that (10) implies (9), so that it is enough to prove (10).

Let us first recall some consequences of Corollary 3.3. Let $E_{t}:=\left\{u_{\lambda}>t\right\}, t \in \mathbb{R}$, and let $\Sigma_{t}$ be its singular set given by Corollary 3.3. Since $f \in L^{\infty}(\Omega)$, around each point $x \in \partial E_{t} \backslash \Sigma_{t}, t \in \mathbb{R}, \partial E_{t}$ is locally the graph of a function in $W^{2, p}$ for all $p \in\left[1, \infty\right.$ ) (hence in $C^{1, \alpha}$ for any $\alpha \in(0,1)$ ). Let $\mathrm{Q}$ be a countable dense set in $\mathbb{R}$ such that $\left\{u_{\lambda}>t\right\}$ is a set of finite perimeter for any $t \in Q$. If we let $\mathcal{N}:=\bigcup_{t \in \mathbb{Q}} \Sigma_{t}$, we then have
$\mathcal{H}^{N-1}(\mathcal{N})=0$.

Since we can write (as in [3])

$$
J_{u_{\lambda}}=\bigcup_{t_{1}, t_{2} \in Q, t_{1}<t_{2}} \partial E_{t_{1}} \cap \partial E_{t_{2}}
$$


in order to prove (10) it suffices to show

$$
t_{2}-t_{1} \leq[f(x)] \quad \mathcal{H}^{N-1} \text {-a.e. on } \partial E_{t_{1}} \cap \partial E_{t_{2}},
$$

for all $t_{1}, t_{2} \in \mathrm{Q}$ with $t_{1}<t_{2}$.

Fix $t_{1}, t_{2} \in \mathbb{Q}$ with $t_{1}<t_{2}$, and let $B_{R}^{\prime}$ be the ball of radius $R>0$ in $\mathbb{R}^{N-1}$ centered at 0 . Let $C_{R}:=B_{R}^{\prime} \times(-R, R)$, and denote $x \in C_{R}$ as $x=(y, z) \in B_{R}^{\prime} \times(-R, R)$. Given $x \in \partial E_{t_{1}} \cap \partial E_{t_{2}} \backslash \mathcal{N}$, by Corollary 3.3 there is $R>0$ such that, after a suitable change of coordinates, we can write the set $\partial E_{t_{i}} \cap C_{R}, i=1,2$, as the graph of a function $v_{i} \in W^{2, p}\left(B_{R}^{\prime}\right)$, $p \in[1, \infty)$, such that $x=\left(0, v_{i}(0)\right) \in C_{R} \subseteq \Omega$ and $\nabla v_{i}(0)=0$. Without loss of generality, we assume that $v_{i}>0$ in $B_{R}^{\prime}$, and that $E_{t_{i}}$ is the supergraph of $v_{i}$. Since $t_{1}<t_{2}$ we have $E_{t_{2}} \subseteq E_{t_{1}}$, which gives in turn $v_{2} \geq v_{1}$ in $B_{R}^{\prime}$. We may also assume that

$$
\mathcal{H}^{N-1}\left(\left\{y \in B_{R}^{\prime}: v_{1}(y)=v_{2}(y)\right\}\right)>0
$$

Notice that, since $\partial E_{t_{i}}$ is of finite $\mathcal{H}^{N-1}$-measure, we may cover $\partial E_{t_{1}} \cap \partial E_{t_{2}} \backslash \mathcal{N}$ by a countable set of such cylinders. By [3, Th. 3.108], for $\mathcal{H}^{N-1}$-a.e. $y \in B_{R}^{\prime}$ the function $f(y, \cdot)$ belongs to $B V((-R, R))$, and the jumps of $f(y, \cdot)$ are the points $z$ such that $(y, z) \in J_{f}$. Recalling that $v_{i}$ is a local minimizer of

$$
\min _{v} \int_{B_{R}^{\prime}} \sqrt{1+|\nabla v|^{2}} d y-\frac{1}{\lambda} \int_{B_{R}^{\prime}} \int_{0}^{v(y)}\left(t_{i}-f(y, z)\right) d z d y=: \mathcal{E}_{i}(v),
$$

by taking a positive smooth test function $\psi(y)$ witn compact support in $B_{R}^{\prime}$, and computing

$$
\lim _{\varepsilon \rightarrow 0^{+}} \frac{\mathcal{E}_{i}(v+\varepsilon \psi)-\mathcal{E}_{i}(v)}{\varepsilon} \geq 0,
$$

we deduce that

$$
\operatorname{div}\left(\frac{\nabla v_{i}(y)}{\sqrt{1+\left|\nabla v_{i}(y)\right|^{2}}}\right)+\frac{1}{\lambda}\left(t_{i}-f^{+}\left(y, v_{i}(y)\right) \leq 0, \quad \mathcal{H}^{N-1} \text {-a.e. in } B_{R}^{\prime} .\right.
$$

In a similar way, we get

$$
\operatorname{div}\left(\frac{\nabla v_{i}(y)}{\sqrt{1+\left|\nabla v_{i}(y)\right|^{2}}}\right)+\frac{1}{\lambda}\left(t_{i}-f^{-}\left(y, v_{i}(y)\right) \geq 0, \quad \mathcal{H}^{N-1} \text {-a.e. in } B_{R}^{\prime} .\right.
$$


Finally we observe that, since $v_{1}, v_{2} \in W^{2, p}\left(B_{R}^{\prime}\right)$ for any $p \in[1, \infty)$ and $v_{2} \geq v_{1}$ in $B_{R}^{\prime}$, by Lemma 3.5 we have that $\nabla v_{1}(y)=\nabla v_{2}(y)$ and $D^{2}\left(v_{1}-v_{2}\right)(y) \leq 0 \mathcal{H}^{N-1}$-a.e. on $\left\{y \in B_{R}^{\prime}: v_{1}(y)=v_{2}(y)\right\}$. Using both inequalities (13) and (14) and Lemma 3.4, it follows that

$$
\begin{aligned}
0<t_{2}-t_{1} & \leq \lambda\left(\operatorname{trace}\left(A\left(\nabla v_{1}(y)\right) D^{2} v_{1}(y)\right)-\operatorname{trace}\left(A\left(\nabla v_{2}(y)\right) D^{2} v_{2}(y)\right)\right) \\
& +f^{+}\left(y, v_{2}(y)\right)-f^{-}\left(y, v_{1}(y)\right) \\
& \leq f^{+}\left(y, v_{2}(y)\right)-f^{-}\left(y, v_{1}(y)\right),
\end{aligned}
$$

$\mathcal{H}^{N-1}$-a.e. on $\left\{y \in B_{R}^{\prime}: v_{1}(y)=v_{2}(y)\right\}$, which gives (11) and concludes the proof.

\section{The jump set of solutions of the Total Variation flow}

Let $\Omega$ be an open bounded set with Lipschitz boundary. We consider the Total Variation flow

$$
\begin{array}{ll}
\frac{\partial u}{\partial t}=\operatorname{div}\left(\frac{D u}{|D u|}\right) & \text { in } Q_{T}=(0, T) \times \Omega, \\
\frac{D u}{|D u|} \cdot v^{\Omega}=0 & \text { in } Q_{T}=(0, T) \times \partial \Omega,
\end{array}
$$

with the initial condition

$$
u(0, x)=f(x), \quad x \in \Omega .
$$

Let us recall that (15) it is the $L^{2}$-gradient flow of the total variation as defined in [9]. In the general case we shall follow [4, 8]. The purpose of this Section is to prove the following result.

Theorem 2. Let $f \in L^{N}(\Omega) \cap B V(\Omega)$. Let $u(t)$ be the solution of (15) with initial condition (16). Then $u(t) \in L^{\infty}(\Omega) \cap B V(\Omega)$ for any $t>0$, and

$$
J_{u(t)} \subseteq J_{u(s)} \subseteq J_{f} \quad \forall t>s>0 .
$$

Moreover

$$
[u(t)] \leq[u(s)] \leq[f] \quad \mathcal{H}^{N-1} \text { a.e. } \forall t>s>0 .
$$

The proof of Theorem 2 is based on the approach in [10] and uses the estimate (10). Let us first recall some basic facts about the operator $-\operatorname{div}\left(\frac{D u}{|D u|}\right)$ in $L^{p}$ spaces. Since it suffices for our purposes, we shall only 

consider the case $p \in\left[\frac{N}{N-1}, \infty\right]$. For any $p \in[1, \infty]$, let us define the
space

$$
X(\Omega)_{p}:=\left\{z \in L^{\infty}\left(\Omega, \mathbb{R}^{N}\right):\left\{\operatorname{div}(\mathrm{z}) \in \mathrm{L}^{\mathrm{p}}(\Omega)\right\} .\right.
$$

If $z \in X(\Omega)_{p}$ and $w \in B V(\Omega) \cap L^{q}(\Omega), p^{-1}+q^{-1}=1$, we define the functional $(z \cdot D w): C_{0}^{\infty}(\Omega) \rightarrow \mathbb{R}$ by the formula

$$
\langle(z \cdot D w), \varphi\rangle:=-\int_{\Omega} w \varphi \operatorname{div} z d x-\int_{\Omega} w z \cdot \nabla \varphi d x .
$$

Then $(z \cdot D w)$ is a Radon measure in $\Omega$, and $(z \cdot D w)=z \cdot \nabla w$ if $w \in W^{1,1}(\Omega) \cap L^{q}(\Omega)$.

Following [7], we observe that for any $z \in X(\Omega)_{p}$ there exists a function $\left[z \cdot v^{\Omega}\right] \in L^{\infty}(\partial \Omega)$ satisfying $\left\|\left[z \cdot v^{\Omega}\right]\right\|_{L^{\infty}(\partial \Omega)} \leq\|z\|_{L^{\infty}\left(\Omega ; \mathbb{R}^{N}\right)}$, and such that for any $u \in B V(\Omega) \cap L^{q}(\Omega)$ we have

$$
\int_{\Omega} u \operatorname{div} z d x+\int_{\Omega}(z \cdot D u)=\int_{\partial \Omega}\left[z \cdot v^{\Omega}\right] u d \mathcal{H}^{N-1} .
$$

Definition 4.1. We define the operator $\mathcal{A}_{p} \subseteq L^{p}(\Omega) \times L^{p}(\Omega)$, with $\frac{N}{N-1} \leq p \leq \infty$, by:

$(u, v) \in \mathcal{A}_{p}$ if and only if $u, v \in L^{p}(\Omega), u \in B V(\Omega)$ and there exists $z \in X(\Omega)_{p}$ with $\|z\|_{\infty} \leq 1$, such that $(z \cdot D u)=|D u|,\left[z \cdot v^{\Omega}\right]=0$ and

$$
v=-\operatorname{div}(z) \quad \text { in } \mathcal{D}^{\prime}(\Omega) \text {. }
$$

By $v \in \mathcal{A}_{p} u$ we mean that $(u, v) \in \mathcal{A}_{p}$. By $L_{w}^{1}((0, T) ; B V(\Omega))$ we denote the space of weakly measurable functions $w:[0, T] \rightarrow B V(\Omega)$ (i.e., the map $t \in[0, T] \rightarrow\langle w(t), \phi\rangle$ is measurable for any $\phi \in B V(\Omega)^{*}$ where $B V(\Omega)^{*}$ denotes the dual of $B V(\Omega)$ ) such that $\int_{0}^{T}\|w(t)\| d t<\infty$.

DeFinition 4.2. A function $u \in C\left([0, T] ; L^{p}(\Omega)\right)$ is a strong solution of (15) if $u \in W_{\mathrm{loc}}^{1,1}\left(0, T ; L^{p}(\Omega)\right) \cap L_{w}^{1}((0, T) ; B V(\Omega))$ and there exists $z \in L^{\infty}\left((0, T) \times \Omega ; \mathbb{R}^{N}\right)$ with $\|z\|_{\infty} \leq 1$ such that

$$
\int_{\Omega}(z(t) \cdot D u(t))=\int_{\Omega}|D u(t)| \text { for a.e. } t>0 .
$$

$$
\left[z(t) \cdot v^{\Omega}\right]=0 \quad \text { in } \partial \Omega \text { for a.e. } t>0 .
$$


and

$$
u_{t}=\operatorname{div} z \quad \text { in } \mathcal{D}^{\prime}((0, T) \times \Omega) .
$$

Proposition 4.3. The operator $\mathcal{A}_{p}$ is $m$-accretive in $L^{p}(\Omega)$, that is for any $f \in L^{p}(\Omega)$ and any $\lambda>0$ there is a unique solution $u \in L^{p}(\Omega)$ of the problem

$$
u+\lambda \mathcal{A}_{p} u \ni f
$$

Moreover, if $u_{1}, u_{2} \in L^{p}(\Omega)$ are the solutions of (21) corresponding to the right hand sides $f_{1}, f_{2} \in L^{p}(\Omega)$, then

$$
\left\|u_{1}-u_{2}\right\|_{p} \leq\left\|f_{1}-f_{2}\right\|_{p} .
$$

Moreover the domain of $\mathcal{A}_{p}$ is dense in $L^{p}(\Omega)$ when $p<\infty$.

We denote by $\mathcal{R}_{\lambda} f$ the solution of (21) and by $\mathcal{R}_{\lambda}^{k} f$ its $k$-iterate, for any $k \geq 1$. We also recall the notion of strong solution for nonlinear semigroups generated by accretive operators.

DEFINITION 4.4. A function $u$ is called a strong solution in the sense of semigroups of

$$
\frac{d u}{d t}+\mathcal{A}_{p} u \ni 0
$$

with $u(0)=f$ if

$$
\left\{\begin{array}{l}
u \in C\left([0, T] ; L^{p}(\Omega)\right) \cap W_{\text {loc }}^{1,1}\left((0, T) ; L^{p}(\Omega)\right) \\
u(t) \in \operatorname{Dom}\left(\mathcal{A}_{p}\right) \quad \text { a.e. in } t>0 \text { and } u^{\prime}+\mathcal{A}_{p} u(t) \ni 0 \text { a.e. } t \in(0, T) \\
u(0)=f .
\end{array}\right.
$$

By Crandall-Ligget's semigroup generation theorem [17], using Proposition 4.3 as in $[6,10]$, one obtains the following result:

THEOREM 3. Let $f \in L^{p}(\Omega)$ if $\frac{N}{N-1} \leq p<\infty$, or $f \in \overline{\operatorname{Dom}\left(\mathcal{A}_{\infty}\right)}$ if $p=\infty$. Then there is a unique strong solution in the sense of semigroups $u(t)=S(t) f:=\lim _{\lambda \downarrow 0, k \lambda \rightarrow t} \mathcal{R}_{\lambda}^{k} f \in C\left([0, T], L^{p}(\Omega)\right)$ of the problem

$$
\frac{d u}{d t}+\mathcal{A}_{p} u \ni 0, \quad u(0)=f .
$$

Moreover, the semigroup solution is a strong solution of (15) and conversely, any strong solution of (15) is a strong solution in the sense of semigroups of (23). 
REMARK 4.5. Notice that given $p \in\left[\frac{N}{N-1}, \infty\right]$ the limit $\lim _{\lambda \downarrow 0, k \lambda \rightarrow t} \mathcal{R}_{\lambda}^{k} f$ is
taken in $L^{p}(\Omega)$.

To prove Theorem 2, we need the following Lemma.

LEMMA 4.6. Let $\left(u_{n}\right)_{n \in \mathbb{N}}$ be a sequence of functions in $B V(\Omega) \cap L^{\infty}(\Omega)$. Assume that $J_{u_{n}} \subseteq J_{u_{0}}$, for all $n \in \mathbb{N}$, and $u_{n} \rightarrow$ u strongly in $L^{\infty}(\Omega)$. Then, $\mathcal{H}^{N-1}$-almost every point of $\Omega \backslash J_{u_{0}}$ is a Lebesgue point for $u$. In particular, if $u \in B V(\Omega)$, then $J_{u} \subseteq J_{u_{0}}$. Moreover, if all the functions $u_{n}$ are continuous at $x \in \Omega$, then also $u$ is continuous at $x$.

Proof. The thesis follows observing that if $x \in \Omega$ is a Lebesgue point for all the functions $u_{n}$, then it is also a Lebesgue point for $u$, and the same is true for a continuity point.

Proof of Theorem 2. We divide the proof into three steps.

Step 1. Assume that $f \in \overline{\operatorname{Dom}\left(\mathcal{A}_{\infty}\right)} \cap B V(\Omega)$. Recall that $\mathcal{R}_{\lambda} f$ denotes the solution of (21), and $\mathcal{R}_{\lambda}^{k} f$ its $k$-iterate, for any $k \geq 1$. In this case, as mentioned in Remark 4.5, we know from [17] that $\mathcal{R}_{\lambda}^{k} f \rightarrow u(t)$ in $L^{\infty}(\Omega)$ when $\lambda \rightarrow 0^{+}$and $k \lambda \rightarrow t$. Then the result follows from Theorem 1 and Lemma 4.6.

Step 2. Let $f \in L^{N}(\Omega)$. By Theorem $3, u \in C\left([0, T] ; L^{N}(\Omega)\right)$. We also know from [6] that $u(t)=S(t) f \in C\left((0, T] ; L^{\infty}(\Omega)\right)$ and $u(t) \in B V(\Omega)$ for any $t>0$. Moreover, the following estimate is a consequence of the 0-homogeneity of the operator $\mathcal{A}_{\infty}[4,6]$

$$
\left\|\frac{d}{d t} S(t) f\right\|_{\infty} \leq 2 \frac{\|f\|_{\infty}}{t} \quad \text { for any } t>0 .
$$

This implies that $u(t) \in \overline{\operatorname{Dom}\left(\mathcal{A}_{\infty}\right)}$ for any $t>0$. Notice that by Step 1 and Theorem 1, we know that

$$
J_{u(t)} \subseteq J_{u(s)} \quad \text { for all } t>s>0 .
$$

Moreover, by (10) we have

$$
[u(t)] \leq[u(s)] \quad \mathcal{H}^{N-1} \text {-a.e. on } J_{u(t)} \text {, for all } t>s>0 .
$$

Step 3. Let $f \in L^{N}(\Omega) \cap B V(\Omega)$. We shall prove that $J_{u(t)} \subseteq J_{f}$ (modulo an $\mathcal{H}^{N-1}$ null set) for any $t>0$. 
Let $Z \subset J_{u(t)}$ be such that $[u(t, \cdot)] \geq \varepsilon>0$ on $Z$ and let $x_{0}$ be a point of $\mathcal{H}^{N-1}$-density 1 in $Z$.

Let us consider $t_{n} \downarrow 0^{+}, t_{n}<t$. By (25) we have $J_{u\left(t_{n}\right)} \subseteq J_{u\left(t_{n-1}\right)}$ and $Z \subseteq J_{u\left(t_{n}\right)}$ for all $n \in \mathbb{N}$ (modulo a $\mathcal{H}^{N-1}$-null set). By (26) we also have

$$
\left[u\left(t_{n}, x\right)\right] \geq[u(t, x)] \geq \varepsilon \quad \mathcal{H}^{N-1} \text {-a.e. on } Z .
$$

Now, observe that

$$
\int_{\Omega}|D u(t)| \leq \int_{\Omega}|D f|
$$

Since

$$
\limsup _{t \rightarrow 0^{+}} \int_{\Omega}|D u(t)| \leq \int_{\Omega}|D f| \leq \liminf _{t \rightarrow 0^{+}} \int_{\Omega}|D u(t)| .
$$

we have that

$$
\lim _{t \rightarrow 0^{+}} \int_{\Omega}|D u(t)|=\int_{\Omega}|D f| .
$$

By Step 2 we know that $u(t) \rightarrow f$ in $L^{N}(\Omega)$. Then $D u(t) \rightarrow D f$ as $t \rightarrow 0+$ weakly* as vector measures in $\Omega$. Since (28) holds, we also have that $|D u(t)| \rightarrow|D f|$ as $t \rightarrow 0+$ weakly* as measures in $\Omega$ ([3], Proposition 1.62).

Let $r>0$ be such that $|D f|\left(\partial B\left(x_{0}, r\right)\right)=0$. By [3, Proposition 1.62] we get

$$
|D f|\left(B\left(x_{0}, r\right)\right)=\lim _{n \rightarrow \infty}\left|D u\left(t_{n}\right)\right|\left(B\left(x_{0}, r\right)\right) \geq \varepsilon \mathcal{H}^{N-1}\left(Z \cap B\left(x_{0}, r\right)\right) .
$$

It follows that, for all points $x_{0}$ of $\mathcal{H}^{N-1}$ density 1 in $Z$, we have

$$
\liminf _{r \rightarrow 0^{+}} \frac{|D f|\left(B\left(x_{0}, r\right)\right)}{\omega_{N-1} r^{N-1}} \geq \varepsilon,
$$

where $\omega_{N-1}$ is the area of the $(N-1)$-dimensional ball. This implies that $Z \subset J_{f}$ (modulo an $\mathcal{H}^{N-1}$ null set). Thus, $J_{u(t)} \subseteq J_{f}$ (modulo an $\mathcal{H}^{N-1}$ null set) for any $t>0$.

REMARK 4.7. Theorem 2 still holds, with analogous proof, in the case of zero Dirichlet boundary conditions or in $\mathbb{R}^{N}[5,8,6]$. Moreover, as in [10] (see also [18]), it also holds for the anisotropic Total Variation flow, when the anisotropy is smooth and elliptic. 
Acknowledgments. V. C. acknowledges partial support by MICINN project reference MTM2009-08171, by GRC reference 2009 SGR 773, and by "ICREA Acadèmia" prize for excellence in research funded both by the Generalitat de Catalunya. M. N. acknowledges partial support by the Fondazione CaRiPaRo Project "Nonlinear Partial Differential Equations: models, analysis, and control-theoretic problems".

\section{REFERENCES}

[1] F. Alter - V. Caselles - A. Chambolle, A characterization of convex calibrable sets in $\mathbb{R}^{N}$. Math. Ann., 332 (2) (2005), pp. 329-366.

[2] L. Ambrosio, Corso introduttivo alla teoria geometrica della misura ed alle superfici minime. Scuola Normale Superiore, Pisa, 1997.

[3] L. Ambrosio - N. Fusco - D. Pallara, Functions of bounded variation and free discontinuity problems. Oxford Mathematical Monographs XVIII, Clarendon Press, 2000.

[4] F. Andreu - C. Ballester - V. Caselles - J. M. Mazón, Minimizing total variation flow. Differential Integral Equations, 14 (3) (2001), pp. 321-360.

[5] F. Andreu - C. Ballester - V. Caselles - J. M. Mazón, The Dirichlet problem for the total variation flow. Journal Functional Analysis, 180 (2001), pp. 347-403.

[6] F. Andreu - V. Caselles - J. M. Mazón, Parabolic Quasilinear Equations Minimizing Linear Growth Functionals. Birkhaüser Verlag, 2004.

[7] G. AnzellotTi, Pairings between measures and bounded functions and compensated compactness. Ann. Mat. Pura Appl., 135 (1983), pp. 293-318.

[8] G. Bellettini - V. Caselles - M. Novaga, The total variation flow in $\mathbb{R}^{N}$. J. Differential Equations, 184 (2) (2002), pp. 475-525.

[9] H. BRÉZIS, Opérateurs maximaux monotones et semi-groupes de contractions dans les espaces de Hilbert. North-Holland Publishing Co., Amsterdam, 1973.

[10] V. Caselles - A. Chambolle - M. Novaga, The discontinuity set of solutions of the TV denoising problem and some extensions. Multiscale modeling \& simulation, 6 (3) (2007), pp. 879-894.

[11] V. Caselles - A. Chambolle - M. Novaga, Total Variation in Imaging. Handbook of Mathematical Methods in Imaging, Springer Verlag, 2010, pp. $1016-1057$.

[12] A. Chambolle - V. Caselles - D. Cremers - M. Novaga - T. Pock, An introduction to Total Variation for Image Analysis. In Theoretical Foundations and Numerical Methods for Sparse Recovery, De Gruyter, Radon Series Comp. Appl. Math., vol. 9 (2010), pp. 263-340.

[13] V. Caselles - A. Chambolle - M. Novaga, Regularity for solutions of the total variation denoising problem. Rev. Mat. Iberoamericana, 27 (1) (2011), pp. 233-252.

[14] A. Chambolle - P.-L. Lions, Image recovery via total variation minimization and related problems. Numer. Math., 76 (1997), pp. 167-188. 
[15] A. Chambolle, An algorithm for mean curvature motion. Interfaces Free Bound., 6 (2) (2004), pp. 195-218.

[16] T. F. Chan - G. H. Golub - P. Mulet, A nonlinear primal-dual method for total variation based image restoration. SIAM J. Sci. Computing, 20 (1999), pp. 1964-1977.

[17] M. G. CRandall - T. M. LigGetT, Generation of Semigroups of Nonlinear Transformations on General Banach Spaces, Amer. J. Math., 93 (1971), pp. $265-298$.

[18] K. JalalzaI, Regularization of inverse problems in image processing. PhD Thesis, École Polytechnique, Palaiseau, Mars 2012.

[19] L. Rudin - S. Osher - E. FAtemi, Nonlinear total variation based noise removal algorithms. Physica D, 60 (1992), pp. 259-268.

[20] W. P. Ziemer, Weakly Differentiable Functions, GTM 120, Springer Verlag, 1989.

Manoscritto pervenuto in redazione il 28 Aprile 2012. 